\title{
NONRESIDUALLY FINITE ONE-RELATOR GROUPS
}

\author{
BY \\ STEPHEN MESKIN
}

\begin{abstract}
The study of one-relator groups includes the connections between group properties and the form of the relator. In this paper we discuss conditions on the form $u^{-1} v^{l} u v^{m}$ which force the corresponding one-relator groups to be nonresidually finite, i.e. the intersection of the normal subgroups of finite index to be nontrivial. Moreover we show that these forms can be detected amongst the words of a free group.
\end{abstract}

\section{Introduction.}

1.1. In order to discuss the one-relator groups which correspond to words of the form $u^{-1} v^{l} u v^{m}$ (where throughout this paper $l$ and $m$ are nonzero integers), indeed for words of any form, it is useful to have a list of the possible word forms which can arise from the given word form when it is cyclically reduced and written without cancellation between factors. Once we have such a list we also have

Theorem A (B. B. Newman, UnPublished; Schupp [7]). There is an algorithm for deciding whether or not an element in a free group has the form $u^{-1} v^{l} u v^{m}$.

For the case $l=-m$, see [9, p. 437]. The list presented here ( $\$ 3)$ is simpler and far shorter than B. B. Newman's original list. The proof in [7] is not directly applicable in our situation. The cyclically reduced forms of $u^{-1} v^{l} u v^{m}$ are then used to prove

THEOREM B. If $u$ and $v$ are any noncommuting elements in a free group, freely generated by a set $X$, and $l$ and $m$ are unequal in absolute value to each other or 1 then $G=\left\langle X ; u^{-1} v^{l} u v^{m}\right\rangle$ is not residually finite.

We will give examples (\$2.3) to show that for some values of $l$ and $m$ the converse of Theorem B is false even when $v$ is not a proper power. It is not known however whether $G$ may be nonresidually finite if $l=-m$ or $l=m \neq \pm 1$.

On the other hand we can show

THEOREM C. $G(l, m)=\left\langle a, b ; a^{-1} b^{l} a b^{m}\right\rangle$ is residually finite if and only if $|l|=1$ or $|m|=1$ or $|l|=|m|$.

This contrasts with the claim made in [2] that $G(l, m)$ is residually finite if one of $l$ or $m$ divides the other.

Received by the editors August 10, 1970.

AMS 1970 subject classifications. Primary 20E25, 20F05; Secondary 20E10, $20 \mathrm{E} 05$.

Key words and phrases. One-relator groups, residually finite, algorithm, free groups, word form.

Copyright (c) 1972, American Mathematical Society 
1.2. I am very grateful to Professor G. Baumslag for suggesting this problem to me and for his help and guidance. Also, I wish to thank B. B. Newman for letting me see his unpublished work and discussing it with me.

\section{General discussion and Theorem $\mathbf{C}$.}

2.1. To show that a group $G$ is not residually finite one must find a nontrivial element of $G$ which is contained in every normal subgroup of finite index.

If $G$ is given by generators $X$ and defining relations $R$ this is equivalent to finding a consequence of $R$ in every finite group which is not a consequence of $R$ in $G$, i.e. which is nontrivial in $G$. There are thus two aspects to the problem: finding an appropriate word and showing that it is nontrivial.

In this section Theorem $\mathrm{C}$ is proven and used to deal with the first aspect of the proof of Theorem B; the second is dealt with in $\$ 4$. Theorem $C$ is useful since the map $a \mapsto u, b \mapsto v$ extends to a homomorphism of $G(l, m)$ into $G$. Hence the image in $G$ of a consequence of $a^{-1} b^{l} a b^{m}$ in a finite group will be a consequence of $u^{-1} v^{l} u v^{m}$ in a finite group. If the homomorphism were actually an embedding, then Theorem B would be a corollary of Theorem C. The embedding question however is still open.

2.2. Theorem $\mathrm{C}$ is a consequence of the following sequence of lemmas.

LEMMA 2.1. If $l$ and $m$ are not powers of the same prime and are unequal in absolute value to each other or 1 then $G(l, m)$ is not residually finite.

Proof. Assume first that $l$ and $m$ do not have the same prime divisors, then we may further assume without loss of generality that there is a prime $p$ dividing $m$ but not $l$. It was shown in [2] that for some such pairs of integers $(l, m)$ the group $G(l, m)$ is non-Hopfian. Indeed it is clear, just as in [2], that $\left[a^{-1} b a, b^{m / p}\right]$ is in the kernel of the ependomorphism induced by the map $a \mapsto a, b \mapsto b^{p}$. It follows from Magnus' theory of one-relator groups [6, \$4.4] (and we will show later in $\S 4$ ) that $\left[a^{-1} b a, b^{m / p}\right] \neq 1$. (Here we use the notational convention $[x, y]=x^{-1} y^{-1} x y$.)

Now the kernel of any ependomorphism of a finitely generated group is contained in every subgroup of finite index. Thus $\left[a^{-1} b a, b^{m / p}\right]$ is a consequence of $a^{-1} b^{l} a b^{m}$ in every finite group.

In the general case, it is clear that $l$ and $m$ have a common divisor $k$ unequal in absolute value to $l$ and $m$ such that $l / k$ and $m / k$ do not have the same prime divisors.

Substituting $b^{k}, l / k$ and $m / k$ for $b, l$ and $m$ in the previous paragraph shows that $\left[a^{-1} b^{k} a, b^{m / p}\right]$ is a consequence of $a^{-1} b^{l} a b^{m}$ in every finite group and as before it is not 1 .

Alternatively $G(l / k, m / k)$ can be embedded in $G(l, m)$ since it is isomorphic to the subgroup of $G(l, m)$ generated by $a$ and $b^{k}$.

If $l$ and $m$ are powers of the same prime then we may use 
LEMMA 2.2. If one of $l$ or $m$ divides the other and they are unequal in absolute value to each other or 1 then $G(l, m)$ is not residually finite.

Proof. It is shown in [4] that for $h=2$ the group

$$
\left\langle x, y, z ; x^{-1} z x=y^{-1} z y=z^{h}\right\rangle
$$

is non-Hopfian. Indeed it is clear, just as in [4], that for $|h|>1,\left[x^{-1} y, z\right]$ is in the kernel of the ependomorphism induced by the map $x \mapsto x, y \mapsto y, z \mapsto z^{h}$. It follows as before that $\left[x^{-1} y, z\right]$ is nontrivial and a consequence of $x^{-1} z x=y^{-1} z y=z^{h}$ in every finite group.

Without loss of generality we may assume that $m=-h l$ where $|h|>1$. Then in $G(l, m)$ we have

$$
a^{-1} b^{l} a=b^{-1} a^{-1} b b^{l} b^{-1} a b=\left(b^{l}\right)^{h} .
$$

Substituting $a, b^{-1} a b$ and $b^{l}$ for $x, y$ and $z$ respectively in the previous paragraph shows as before that $\left[a, b, b^{l}\right]$ is nontrivial and a consequence of $a^{-1} b^{l} a b^{m}$ in every finite group.

Alternatively the group in the first paragraph can be embedded in $G(l, m)$. This follows from the fact that the normal subgroup generated by $a$ and $b^{l}$ has the presentation

$$
\left\langle a_{0}, a_{1}, \ldots, a_{m-1}, b_{*} ; a_{i}^{-1} b_{*} a_{i}=b_{*}^{n}, i=0,1, \ldots, m-1\right\rangle
$$

where $a_{i}=b^{i} a b^{-i}$ and $b_{*}=b^{l}$.

From the proofs of Lemmas 2.1 and 2.2 we can extract information which will be useful in the proof of Theorem B.

COROLlaRy 2.3. If $l$ and $m$ are unequal in absolute value to each other or 1 then there exist integers $\lambda$ and $\mu$ not divisible by $l$ and $m$ respectively such that $\left[a, b^{\lambda}, b^{\mu}\right]$ is a consequence of $a^{-1} b^{l} a b^{m}$ in every finite group.

Proof. If $l$ and $m$ are not powers of the same prime then by Lemma 2.1 there exist integers $k$ and $p$ such that $-k$ and $m / p$ can be chosen for $\lambda$ and $\mu$. If $l$ and $m$ are powers of the same prime then, by Lemma 2.2, we can then choose 1 and $l$.

LEMMA 2.4. If $|l|=1$ or $|m|=1$ then $G(l, m)$ is residually finite.

Proof. This follows from [3, Theorem 1] since if $|l|=1$ or $|m|=1$ then $G(l, m)$ is finitely generated and metabelian.

LEMMA 2.5. If $|l|=|m|$ then $G(l, m)$ is residually finite.

Proof. Let $A$ be the normal subgroup generated by $a$ and $b^{m}$ and let $B$ be the subgroup generated by $b^{m}$. Then $B$ is normal and $A / B$ is free. Thus $A$ is residually finite; for Mal'cev has shown that the semidirect extension of a finitely generated residually finite group by a residually finite group is again residually finite [5, p. 506]. Now $G(l, m) / A$ is finite and so $G(l, m)$ is residually finite. 
Alternatively $G(l, m)$ may be viewed as the generalized free product of $\mathrm{gp}\left(a, b^{m}\right)$ and $\mathrm{gp}(b)$, in which case the result follows from [1, Theorem 8 or 9], or [8, Corollary 3.4].

2.3. One would naturally suspect the converse of Theorem $B$ to be false, i.e. even though $l$ and $m$ are equal in absolute value to each other or 1 there may exist $u$ and $v$ such that $\left\langle X ; u^{-1} v^{l} u v^{m}\right\rangle$ is not residually finite. However when $l$ and $m$ are equal in absolute value to each other, the author knows of no example except for $l=m= \pm 1$. Indeed let $u=v_{*}^{n}$ and $v=v_{*}^{-n} u_{*}^{-1} v_{*} u_{*}$, so that $u^{-1} v u v=v_{*}^{-2 n} u_{*}^{-1} v_{*}^{2} u_{*}$ whence $\left\langle X ; u^{-1} v u v\right\rangle=\left\langle X ; u_{*}^{-1} v_{*}^{-2} u_{*} v_{*}^{-2 n}\right\rangle$. Thus Theorem $\mathrm{B}$ itself yields many examples. Theorem $\mathbf{B}$ also yields many examples of nonresidually finite groups with $l=1$ and $|m|>1$. However, in all these examples $v$ is a proper power.

For examples without this defect, consider the groups

$$
\left\langle a, b ; b^{-1} a^{-m} b a b^{-1} a^{m} b a^{-m}\right\rangle \text {. }
$$

Here we let $u=b^{-1} a^{m} b$ (or $\left[a^{m}, b\right]$ ) and $v=a$. They are not residually finite since $[a, b, a, a]$ is a consequence of the relator in every finite group. Indeed, if $a$ has finite order $n$, then

$$
a=b^{-1} a^{-m n} b a b^{-1} a^{m n} b=a^{m^{n}}
$$

thus

$$
[a, b, a]=b^{-1} a^{-1} b a^{-1} b^{-1} a b a=b^{-1} a^{-m^{n}} b a^{-1} b^{-1} a^{m^{n}} b a=a^{1-m^{m^{n-1}}}
$$

and so $[a, b, a]$ commutes with $a$. It is easy to see that $[a, b, a, a] \neq 1$ in the group itself.

3. Theorem A. Theorem A is an obvious consequence of the following lemma.

LEMMA 3.1. Let $w$ be an element in a free group $F$. Then there exist noncommuting elements $u$ and $v$ in $F$ such that $w=u^{-1} v^{l} u v^{m}$ iff there exist elements $r, s, t, x, y, z$ such that some cyclically reduced conjugate of $w$ is equal to one of the products below (which are assumed to be cyclically reduced as written):

(i) $z^{-1}(x y)^{|l|} z(y x)^{|m|} ; x y z x^{-1} y^{-1} z^{-1} \neq 1 ; \operatorname{lm}>0$.

(ii) $\left(x z^{-1} y z\right)^{|l|-1} x z^{-1} y^{2} z x\left(z^{-1} y z x\right)^{|m|-1} ; x z^{-1} y z x^{-1} z^{-1} y^{-1} z \neq 1 ; \operatorname{lm}>0$.

(iii) $z^{-1}(x y)^{|l|} z\left(x^{-1} y^{-1}\right)^{|m|} ; x y z x^{-1} y^{-1} z^{-1} \neq 1 ; \operatorname{lm}<0$.

(iv) $(x y)^{|l|-1} s t^{-1}\left(x^{-1} y^{-1}\right)^{|m|-1} ; x y=s r ; y x=t r ; x y x^{-1} y^{-1} \neq 1 ; \operatorname{lm}<-1$.

Proof. If some conjugate of $w$ is equal to one of the products (i)-(iv) and the associated conditions are satisfied then clearly there exist noncommuting elements $u$ and $v$ such that $w=u^{-1} v^{l} u v^{m}$. For example suppose $g^{-1} w g=z^{-1}(x y)^{l} z(y x)^{m}$, $x y z x^{-1} y^{-1} z^{-1} \neq 1, l>0$ and $m>0$. Then with $u=g y z g^{-1}$ and $v=g y x g^{-1}$, we have $w=u^{-1} v^{l} u v^{m}$ and $v u v^{-1} u^{-1}=g y\left(x y z x^{-1} y^{-1} z^{-1}\right) y^{-1} g^{-1} \neq 1$.

Conversely suppose $w=u^{-1} v^{l} u v^{m}$ for noncommuting $u$ and $v$, then with $x=v^{ \pm 1}$, $y=1$ and $z=u, w$ satisfies (i) or (iii) depending on the values of $l$ and $m$. The product however may not be cyclically reduced as written. We will show that any 
product of the form of one of (i)-(iv) which is not cyclically reduced is conjugate to a shorter product satisfying one of (i)-(iv). Since the lemma holds vacuously for products of length 0 , the result will follow by induction.

For technical reasons a product will be assigned two lengths, $L_{1}$ and $L_{2}$, which differ only for products of type (iv). We say that the product $\bar{p}$ is shorter than the product $p$ if $L_{1}(\bar{p})<L_{1}(p)$ or $L_{1}(\bar{p})=L_{1}(p)$ and $L_{2}(\bar{p})<L_{2}(p)$. Now $L_{2}(p)$ is just the length of $p$ as written, i.e. before cancellation between its factors is taken into account. For example if $p$ is a product of type (iv) then

$$
L_{2}(p)=(|l|+|m|-2)(|x|+|y|)+|s|+|t| .
$$

In this expression, $|\cdot|$ denotes the usual length of a word in a free group. If $p$ is a product of type (iv) then we define $L_{1}(p)=(|l|+|m|)(|x|+|y|)$ otherwise $L_{1}(p)$ $=L_{2}(p)$. We assume throughout the argument that $r, s, t, x, y$ and $z$ are freely reduced.

Cyclic cancellation between the factors of one of the products we are considering may occur either between factors which are obviously adjacent, including the terminal and initial factors, or between factors whose adjacency arises because the intervening factors are one. We will begin with cancellations of the first type. In all but one of these cases the product resulting after cancellation is of the same type as the original. Cancellations of the second kind can for the most part be dealt with by referring to those previously discussed. The symbols representing the new product and the factors therein are the old symbols with bars above them.

Suppose first of all that there is cancellation at either $z^{-1} x, y x$ or $y z$ in a product of type (i) or (iii). Then at least two of the words $x, y^{-1}$ and $z$ have a common initial segment; call it $c$. Thus there exist $\bar{x}, \bar{y}$ and $\bar{z}$ such that there is no cancellation in at least two of the factorizations:

$$
x=c \bar{x}, \quad y^{-1}=c \bar{y}^{-1} \text { and } z=c \bar{z} .
$$

It is clear that by substitution we get a new product $\bar{p}$ of the same type, that $\bar{p}=p$ and that $x y z x^{-1} y^{-1} z^{-1}=c\left(\bar{x} \bar{y} \bar{z} \bar{x}^{-1} \bar{y}^{-1} \bar{z}^{-1}\right) c^{-1}$. Moreover at least two of the equations $|x|=|c|+|\bar{x}|,|y|=|c|+|\bar{y}|$ and $|z|=|c|+|\bar{z}|$ hold, in any case

$$
|\xi| \leqq|c|+|\xi| \text { for } \xi=x, y \text { and } z \text {. }
$$

Thus if $|z|=|c|+|\bar{z}|$, then $|\bar{x}|+|\bar{y}| \leqq|x|+|y|$ and hence

$$
\begin{aligned}
L_{1}(\bar{p})+2|c| & \leqq(|l|+|m|)(|\bar{x}|+|\bar{y}|)+2(|\bar{z}|+|c|) \\
& =(|l|+|m|)(|x|+|y|)+2|z|=L_{1}(p) .
\end{aligned}
$$

On the other hand, if $|z| \neq|c|+|\bar{z}|$, then

$$
\begin{aligned}
L_{1}(\bar{p})+2(|l|+|m|-1)|c| & =(|l|+|m|)(|\bar{x}|+|c|+|\bar{y}|+|c|)+2(|\bar{z}|-|c|) \\
& \leqq(|l|+|m|)(|x|+|y|)+2|z|=L_{1}(p) .
\end{aligned}
$$

Since $|l|+|m|>1$ we have $L_{1}(\bar{p})<L(p)$ in either case. 
Suppose now that there is cancellation at either $x z^{-1}, x y$ or $z y$ in a product of type (i) or (iii). Then at least two of the words $x, y^{-1}$ and $z$ have a common terminal segment; call it $c$. The argument proceeds now exactly as in the previous paragraph except that in this case $\bar{p}=c p c^{-1}$.

Suppose now that there is cancellation at either $z^{-1} y, y y$ or $y z$ in a product of type (ii). Then at least two of the words $y, y^{-1}$ and $z$ have a common initial segment; call it $c$. Thus there exist $\bar{y}$ and $\bar{z}$ such that there is no cancellation in at least two of the factorizations: $y=c \bar{y} c^{-1}, z=c \bar{z}$. Let $x=\bar{x}$. It is clear that by substitution we get a new product $\bar{p}$ of the same type, that $\bar{p}=p$ and that

Moreover either

$$
x z^{-1} y z x^{-1} z^{-1} y^{-1} z=\bar{x} \bar{z}^{-1} \bar{y} \bar{z} \bar{x}^{-1} \bar{z}^{-1} \bar{y}^{-1} \bar{z} \text {. }
$$

$$
|y|=2|c|+|\bar{y}| \text { and }|\bar{z}| \leqq|c|+|z| \text { or }|y| \geqq|\bar{y}| \text { and }|z|=|\bar{z}|+|c| \text {. }
$$

In the first instance

$$
\begin{aligned}
L_{1}(\bar{p})+2|c| & =(|l|+|m|)(|\bar{x}|+|\bar{y}|+2|c|)+2(|l|+|m|-1)(|\bar{z}|-|c|) \\
& \leqq(|l|+|m|)(|x|+|y|)+2(|l|+|m|-1)|z|=L_{1}(p),
\end{aligned}
$$

and hence we have $L_{1}(\bar{p})<L_{1}(p)$ in either case.

Suppose now that there is cancellation at either $x z^{-1}, x x$ or $z x$ in a product of type (ii). Then at least two of the words, $x, x^{-1}$ and $z$ have a common terminal segment; call it $c$. The argument proceeds now exactly as in the previous paragraph except that in this case, $\bar{p}=c p c^{-1}$.

If $z=1$ in a product of type (i) or type (ii) the resulting products are alike. The new possible cancellations for a product of type (i) are at $y y$ and $x x$ which have already been discussed as a possible cancellation in a product of type (ii). Similarly for type (ii) the new ones are at $x y$ and $y x$ which have been covered already for type (i). If $x=1$ or $y=1$ in a product of type (i) or type (iii) the new possible cancellations are handled by relabeling $y$ as $x$ or $x$ as $y$ and referring again to the previous discussion.

If $x=1$ or $y=1$ in a product of type (ii), then $x z^{-1} y z x^{-1} z^{-1} y^{-1} z=1$ and so this case does not arise. If $z=1$ in a product of type (iii) and $l m=-1$ then the new possible cancellations are handled as before by relabeling $x$ as $z^{-1}$. However if lm $<-1$ then the product is one of type (iv) with $r=1$. This completes our discussion of products of type (i), (ii) and (iii).

In considering products of type (iv) we may assume

(1) that there is no cancellation at $x y$ and $y x$. For if there were we could write the product as a product of type (iii) for which we have already handled these cancellations. Although in this process the actual $\left(L_{2}\right)$ length may be increased, we will have definitely decreased $L_{1}$, which is all that is required.

Suppose now that there is cancellation at $s t^{-1}$. Thus $s=\bar{s} c$ and $t=\bar{t} c$. Let $\bar{r}=c r$, $\bar{x}=x, \bar{y}=y$ and $\bar{p}=p$. Then

$$
L_{1}(\bar{p})=L_{1}(p) \text { and } L_{2}(\bar{p})+2|c|=L_{2}(p) .
$$


We can assume henceforth

(2) that there is no cancellation at $s t^{-1}$;

and for the time being

(3) that $s \neq 1$ and $t \neq 1$.

By (2) there can be cancellation at no more than one of $s r$ and $t r$. By (1) we have

$$
|s r|=|x|+|y|=|t r| \text {. }
$$

Thus neither $s$ nor $t$ may cancel completely against $r$. For suppose $s$ did then it would follow from (3) that

$$
|s r|=|r|-|s|<|r|+|t|=|t r|,
$$

which is a contradiction. It follows that $s$ and $t$ have initial segments in common with $x$ and $y$ respectively. Hence by (1) there can be no cancellation at $y s$ and $x t$. Furthermore if there is cancellation at $y^{-1} x$ then $x, y, s$ and $t$ all have a common initial segment; call it $c$. Let $x=c \bar{x} c^{-1}, y=c \bar{y} c^{-1}, s=c \bar{s}, t=c \bar{t}, r=\bar{r} c^{-1}$ and $\bar{p}=c^{-1} p c$. Then $L_{1}(\bar{p}) \leqq L_{1}(p)$ and $L_{2}(\bar{p})+2|c| \leqq L_{2}(p)$.

Since $x y \neq y x$ we cannot have both $s=1$ and $t=1$. Suppose $s=1$ (if $t=1$ we can use a similar argument) then

$$
|r|=|x|+|y|=|t r|>|r|-|t| \text {. }
$$

Hence some but not all of $t$ cancels at $t r$ so that we can write $t=\bar{t} \bar{s}^{-1}$ and $r=\bar{s} \bar{r}$ where $\bar{t} \neq 1$ and $\bar{s} \neq 1$. Clearly $x y=\bar{s} \bar{r}$ and $y x=\bar{t} \bar{r}$ and the lengths are not altered by the new notation, so we are back to our previous discussion.

Although the proof of the lemma is now complete, we must make an additional comment in order to use it in the next section. At each step in the reduction process outlined in the proof, a product $p$ is conjugated into a new product $\bar{p}$. We can find $u, v, \bar{u}$ and $\bar{v}$ such $p=u^{-1} v^{l} u v^{m}$ and $\bar{p}=\bar{u}^{-1} \bar{v}^{l} \bar{u} \bar{v}^{m}$, however given $u$ and $v$ we cannot always cloose $\bar{u}$ and $\bar{v}$ to be the images of $u$ and $v$ under the conjugation. We can, on the other hand, except when $\operatorname{lm}=-1$, choose $\bar{u}$ and $\bar{v}$ so that $\bar{u}^{-1} \bar{v} \bar{u}$ and $\bar{v}$ are the images of $u^{-1} v u$ and $v$ respectively. In particular $\left[\bar{u}, \bar{v}^{\lambda}, \bar{v}^{\mu}\right]$ will be the image of $\left[u, v^{\lambda}, v^{\mu}\right]$ under the same conjugation which takes $p$ to $\bar{p}$.

4. Theorem B. The proof of Theorem B may now be completed (see Corollary 2.3) by proving

LEMMA 4.1. If $u$ and $v$ are any noncommuting elements in a free group, freely generated by a set $X$ and $G=\left\langle X ; u^{-1} v^{l} u v^{m}\right\rangle$, then for all $\lambda$ and $\mu$ not divisible by $l$ and $m$ respectively $\left[u, v^{\lambda}, v^{\mu}\right] \neq 1$.

Proof. We may assume without loss of generality that $l \geqq|m|>1$ and by Lemma 3.1 that there exists $x, y, \ldots$ such that $u^{-1} v^{l} u v^{m}=p$ and $p$ is cyclically reduced, where one of the following is valid:

(i) $u=x^{-1} z, v=y x, m>0$ and $p=z^{-1}(x y)^{l} z(y x)^{m}$.

(ii) $u=x^{-1}, v=z^{-1} y z x, m>0$ and $p=\left(x z^{-1} y z\right)^{l-1} x z^{-1} y^{2} z x\left(z^{-1} y z x\right)^{m-1}$. 
(iii) $u=x^{-1} z, v=y x, m<0$ and $p=z^{-1}(x y)^{l} z\left(x^{-1} y^{-1}\right)^{-m}$.

(iv) $u=x^{-1}, v=y x=t r, x y=s r, m<0$ and $p=(x y)^{l-1} s t^{-1}\left(x^{-1} y^{-1}\right)^{-m-1}$.

Our proof, as with most proofs in the theory of one relator groups, is modeled after that of the Freiheitssatz [6, p. 252 et seq.]. Thus we assume inductively that the lemma is valid for all $p^{*}$ with $L_{2}\left(p^{*}\right)<L_{2}(p)$. The lemma holds vacuously for products of length 0 . Furthermore we assume for the moment that there exists a generator $a \in X$ which appears in $v$ such that the exponent sum of $a$ in $v$ is zero, i.e. $\sigma_{a}(v)=0$. It follows also that $\sigma_{a}(p)=0$.

We then consider the Magnus subgroup, $M$, generated by

$$
\left\{a^{-i} b a^{i}, a^{-i} c a^{i}, \ldots ; i=0, \pm 1, \ldots\right\}
$$

where $X=\{a, b, c, \ldots\} . M$ is the normal subgroup of $G$ generated by $\{b, c, \ldots\}$ and $m \in M$ if and only if $\sigma_{a}(m)=0$. If $m \in M$ we let $m_{i}=a^{-i} m a^{i}$ and assume that $m_{i}$ is written as a word in the generators $\left\{\ldots, b_{-1}, b_{0}, b_{1}, \ldots ; \ldots, c_{-1}, c_{0}, c_{1}, \ldots\right\}$. Since $\sigma_{a}(p)=0$ some generator other than $a, b$ say, appears in $p$ and, for some $i, b_{i}$ appears in $p_{0}$. If $\alpha$ and $\beta$ are respectively the least and greatest of the indices $i$ such that $b_{i}$ appears in $p_{0}$,

$$
X_{0}=\left\{b_{\alpha}, b_{\alpha+1}, \ldots, b_{\beta} ; \ldots, c_{-1}, c_{0}, c_{1}, \ldots ; \ldots\right\}
$$

and $G_{0}=\operatorname{gp}\left\{X_{0}\right\}$, then $G_{0}=\left\langle X_{0} ; p_{0}\right\rangle$ and moreover $L_{2}\left(p_{0}\right)<L_{2}(p)$.

We now analyze the structure of $G_{0}$, show that it contains $\left[u, v^{\lambda}, v^{\mu}\right]$ and that it is not 1.

Cases (i) and (iii). Let $\sigma_{a}(u)=n$ and $\sigma_{a}(y)=k$. We may assume $n \geqq 0$. It follows that $\sigma_{a}(x)=-k$ and $\sigma_{a}(z)=n-k$. Hence $y=y_{0} a^{k}, x=a^{-k} x_{0}, z=a^{n-k} z_{0}, v=y x=y_{0} x_{0}$, $u=x^{-1} z=a^{n} x_{n}^{-1} z_{0}$ and $p_{0}=z_{0}^{-1}\left(x_{n} y_{n}\right)^{l} z_{0}\left(y_{0} x_{0}\right)^{m}$ in Case(i) or $z_{0}^{-1}\left(x_{n} y_{n}\right)^{l} z_{0}\left(x_{0}^{-1} y_{0}^{-1}\right)^{-m}$ in Case (iii). In either case

$$
\begin{aligned}
{\left[u, v^{\lambda}, v^{\mu}\right] } & =\left[a^{n} x_{n}^{-1} z_{0},\left(y_{0} x_{0}\right)^{\lambda},\left(y_{0} x_{0}\right)^{\mu}\right] \\
& =\left[z_{0}^{-1}\left(x_{n} y_{n}\right)^{-\lambda} z_{0}\left(y_{0} x_{0}\right)^{\lambda},\left(y_{0} x_{0}\right)^{\mu}\right] .
\end{aligned}
$$

Now if $n=0$, we are done by induction.

If $n>0$, we let $N=$ normal subgroup of $G_{0}$ generated by $\left(x_{n} y_{n}\right)^{l}$; then

$$
G_{0} / N=\left\langle X_{0} ;\left(x_{n} y_{n}\right)^{l},\left(y_{0} x_{0}\right)^{m}\right\rangle .
$$

We might as well have assumed that $b$ appeared in $v$ and thus that $b_{i}$ appears in $y_{0} x_{0}$ for some $i$. If $\rho$ and $\sigma$ are respectively the least and greatest of the indices $i$ such that $b_{i}$ appears in $y_{0} x_{0}, \tau=\min (\sigma+1, \rho+n)$,

$$
\begin{aligned}
& Y_{\alpha}=\left\{b_{\alpha}, b_{\alpha+1}, \ldots, b_{\sigma} ; \ldots, c_{-1}, c_{0}, c_{1}, \ldots ; \ldots\right\}, \\
& Y_{\beta}=\left\{b_{\imath}, b_{\imath+1}, \ldots, b_{\beta} ; \ldots, c_{-1}, c_{0}, c_{1}, \ldots ; \ldots\right\}, \\
& Y_{\tau}=\left\{b_{\imath}, \ldots, b_{\sigma} ; \ldots, c_{-1}, c_{0}, c_{1}, \ldots ; \ldots\right\},
\end{aligned}
$$

and $H_{\theta}=\operatorname{gp}\left(Y_{\theta}\right)$ in $G / N$ for $\theta=\alpha, \beta$ or $\tau$, then

$$
H_{\alpha}=\left\langle Y_{\alpha} ;\left(y_{0} x_{0}\right)^{m}\right\rangle, \quad H_{\beta}=\left\langle Y_{\beta} ;\left(x_{n} y_{n}\right)^{l}\right\rangle, \quad H_{\tau}=\left\langle Y_{\tau} ;\right\rangle,
$$

and moreover $G_{0} / N$ is the generalized free product of $H_{\alpha}$ and $H_{\beta}$ amalgamating $H_{\tau}$. 
Since $\lambda$ and $\mu$ are not divisible by $l$ and $m$ respectively, $\left(x_{n} y_{n}\right)^{\lambda}$ and $\left(y_{0} x_{0}\right)^{\mu}$ both have finite order but are not 1 in $G_{0} / N$ and hence none of their conjugates lie in the amalgamated subgroup $H_{\tau}$, which is free. Consequently $\left[u, v^{\lambda}, v^{\mu}\right]$ has length at least four in the generalized free product $G_{0} / N$ and so cannot be 1 .

Case (ii). Let $\sigma_{a}(u)=n$ and $\sigma_{a}(z)=\dot{k}$. We may assume $n \geqq 0$. It follows that $\sigma_{a}(x)=-n$ and $\sigma_{a}(y)=n$. Hence $y=a^{k} y_{0} a^{n-k}, x=a^{-n} x_{0}, z=a^{k} z_{0}, v=z^{-1} y z x$ $=z_{0}^{-1} y_{0} z_{-n} x_{0}, u=x^{-1}=x_{0}^{-1} a^{n}$ and

$$
p_{0}=\left(x_{n} z_{n}^{-1} y_{n} z_{0}\right)^{l-1} x_{n} z_{n}^{-1} y_{n} y_{0} z_{-n} x_{0}\left(z_{0}^{-1} y_{0} z_{n} x_{0}\right)^{m-1} \text {. }
$$

Moreover

$$
\begin{aligned}
{\left[u, v^{\lambda}, v^{\mu}\right] } & =\left[x_{0}^{-1} a^{n},\left(z_{0}^{-1} y_{0} z_{-n} x_{0}\right)^{\lambda},\left(z_{0}^{-1} y_{0} z_{-n} x_{0}\right)^{\mu}\right] \\
& =\left[\left(x_{n} z_{n}^{-1} y_{n} z_{0}\right)^{-\lambda}\left(z_{0}^{-1} y_{0} z_{-n} x_{0}\right)^{\lambda},\left(z_{0}^{-1} y_{0} z_{-n} x_{0}\right)^{\mu}\right] .
\end{aligned}
$$

Now if $n=0$, we are done by induction.

If $n>0$, then

$$
G_{0}=\left\langle X_{0} ;\left(x_{n} z_{n}^{-1} y_{n} z_{0}\right)^{l}=\left(z_{0}^{-1} y_{0} z_{-n} x_{0}\right)^{m}\right\rangle .
$$

We may proceed exactly as in the previous case by considering $G_{0} / N$ where $N$ is the normal subgroup of $G_{0}$ generated by $\left(x_{n} z_{n}^{-1} y_{n} z_{0}\right)^{l}$. However, in this case $G_{0}$ is already a generalized free product this time of two free groups and again an argument similar to the previous one can be used.

Case (iv). Let $\sigma_{a}(u)=n$ and $\sigma_{a}(t)=k$. We may assume $n \geqq 0$. It follows that $\sigma_{a}(x)=-n, \quad \sigma_{a}(y)=n, \quad \sigma_{a}(r)=-k$ and $\sigma_{a}(s)=k$. Hence $y=y_{0} a^{n}, x=a^{-n} x_{0}$, $t=t_{0} a^{k}, \quad s=s_{0} a^{k}, \quad r=a^{-k} r_{0}, \quad v=y x=y_{0} x_{0}=t r=t_{0} r_{0}, \quad u=x^{-1}=x_{0}^{-1} a^{n}, \quad p_{0}=$ $\left(x_{n} y_{n}\right)^{l-1} s_{0} t_{0}^{-1}\left(x_{0}^{-1} y_{0}^{-1}\right)^{-m-1}$, and

$$
\begin{aligned}
{\left[u, v^{\lambda}, v^{\mu}\right] } & =\left[x_{0}^{-1} a^{n},\left(y_{0} x_{0}\right)^{\lambda},\left(y_{0} x_{0}\right)^{\mu}\right] \\
& =\left[\left(x_{n} y_{n}\right)^{-\lambda}\left(y_{0} x_{0}\right)^{\lambda},\left(y_{0} x_{0}\right)^{\mu}\right] .
\end{aligned}
$$

Now if $n=0$, we are done by induction.

If $n>0$, note that $r_{0} \in \mathrm{gp}\left(X_{0}\right)$ so that

$$
G_{0}=\left\langle X_{0} ;\left(x_{n} y_{n}\right)^{l}=\left(y_{0} x_{0}\right)^{-m}\right\rangle .
$$

We may proceed now as in Case (ii).

Finally we deal in the standard way with the possibility that $\sigma_{x}(v) \neq 0$ for all $x \in X$ which appear in $p$. Since $u$ and $v$ do not commute there must be at least two distinct elements $a$ and $b$ of $X$ which appear in $p$ and hence also in $v$ with nonzero exponent sum. Let $\sigma_{a}(v)=\alpha$ and $\sigma_{b}(v)=\beta$. Then the map $a \rightarrow A^{\beta}, b \rightarrow B A^{-\alpha}$, $c \rightarrow C, \ldots$ extends to an embedding of $G$ into

$$
H=\left\langle A, B, C, \ldots ; U^{-1} V^{l} U V^{m}\right\rangle
$$

when $U$ and $V$ are the images of $u$ and $v$. Now $\sigma_{A}(V)=0$ and, although the length of the new relator is longer than that of $p$, when we consider as we did before $H_{0}$, the length of the relator in $H_{0}$ will be shorter than that of $p$. Thus by the previous argument, the image of $\left[u, v^{\lambda}, v^{\mu}\right]$ is not 1 and thus neither is $\left[u, v^{\lambda}, v^{\mu}\right]$ itself. 


\section{REFERENCES}

1. Gilbert Baumslag, On the residual finiteness of generalised free products of nilpotent groups, Trans. Amer. Math. Soc. 106 (1963), 193-209. MR 26 \#2489.

2. Gilbert Baumslag and Donald Solitar, Some two-generator one-relator non-Hopfian groups, Bull. Amer. Math. Soc. 68 (1962), 199-201. MR 26 \#204.

3. P. Hall, On the finiteness of certain soluble groups, Proc. London Math. Soc. (3) 9 (1959), 595-622. MR 22 \#1618.

4. Graham Higman, A finitely related group with an isomorphic proper factor group, J. London Math. Soc. 25 (1951), 59-61. MR 12, 390.

5. A. G. Kuroš, Theory of groups, 3rd ed., "Nauka”, Moscow, 1967; English transl. of 2nd ed., Chelsea, New York, 1955. MR 17, 124; MR 40 \#2740.

6. W. Magnus, A. Karrass and D. Solitar, Combinatorial group theory: Presentations of groups in terms of generators and relations, Pure and Appl. Math., vol. 13, Interscience, New York, 1966. MR 34 \#7617.

7. Paul E. Schupp, On the substitution problem for free groups, Proc. Amer. Math. Soc. 23 (1969), 421-423. MR 39 \#6963.

8. Peter Stebe, Residual finiteness of a class of knot groups, Comm. Pure Appl. Math. 21 (1968), 563-583. MR 38 \#5902.

9. M. J. Wicks, Commutators in free products, J. London Math. Soc. 37 (1962), 433-444. MR 26 \#179.

Department of Mathematics, Institute of Advanced Studies, Australian National University, CaNBerra, ACT. 2600, Australia

Current address: Department of Mathematics, University of Connecticut, Storrs, Connecticut 06268 